\title{
Effects of early breastfeeding education on maintenance of breastfeeding practice: A prospective observational study
}

\author{
Tae-Hee Kim ${ }^{1}$, Hae-Hyeog Lee ${ }^{1}$, Junsik Park ${ }^{1}$, Seyeon Jang ${ }^{1}$, Minjung Kim ${ }^{2}$ \\ ${ }^{1}$ Department of Obstetrics and Gynecology, College of Medicine, Soonchunhyang University, Bucheon, South Korea \\ ${ }^{2}$ Department of Food and Nutrition, Hanyang University, Seoul, South Korea \\ Email: "hhl22@chol.com, ${ }^{*}$ hhl22@schmc.ac.kr
}

Received 20 March 2013; revised 21 April 2013; accepted 15 May 2013

Copyright (c) 2013 Tae-Hee Kim et al. This is an open access article distributed under the Creative Commons Attribution License, which permits unrestricted use, distribution, and reproduction in any medium, provided the original work is properly cited.

\begin{abstract}
Background: Breast feeding is an ideal feeding type for most infants and mothers. We evaluated the effect of early breast-feeding and prenatal education of breastfeeding in maintenance of breast-feeding practice after delivery. Methods: Eighty two pregnant women who experienced vaginal delivery were recruited from the department of obstetrics and gynecology of a university hospital between January 2006 and October 2006. These differences among variables were determined using SPSS ver. 10.0 (SPSS Inc., Chicago, IL, USA). Follow-up telephone interviews were conducted 1 week and 4 weeks after delivery to check feeding method for baby and other complications. Results: We calculated the correlations among the pregnant women's age, height, and weight, abortion experience, the newborn's weight, and social factors using ANOVA. There was no statistically significant relationship between early breast-feeding and maintenance of breastfeeding practice. Conclusions: Antenatal and postpartum period education about breast-feeding is necessary for breast-feeding practice maintenance. Further studies in developing educational programs are needed.
\end{abstract}

Keywords: Breastfeeding; Education; Postpartum Period

\section{INTRODUCTION}

Breast-feeding after delivery has gone through various changes due to continuous westernization and family structure change to nuclear family. Whereas breast-feeding in Europe reached $75 \%$, the United States $52 \%$ and $45 \%$ in Japan [1], South Korea witnessed a sharp decrease in breast-feeding from 95\% in 1961 to $10.2 \%$ in 2000 [2].

\footnotetext{
"Corresponding author.
}

Breast milk not only contains high level of anti-bacterial and anti-virus antibodies including Immunoglobulin A, but also its abundance in macrophage, iron binding protein and others allows greater resistance against gastrointestinal and respiratory infection [3]. However, despite such advantages, the rate of breast-feeding is at continuous decline. For successful adoption of breastfeeding, acknowledging its benefits, mothers' willingness and most importantly systematic education on breastfeeding are necessary.

Accordingly, in an effort to promote breast-feeding practice and provide an appropriate foundation for developing a prenatal educational program, we analyzed how early breast-feeding to the new-born infants affects postpartum breast-feeding practice and studied the effects of education on breast-feeding practice.

\section{MATERIALS AND METHODS}

This study is based on the surveys from 82 primipara pregnant women who experienced vaginal delivery at a university hospital of obstetrics and gynecology department during the period from January 2006 to October in 2006. These 82 selected women had no medical and surgical complications prior to delivery, nor had peculiarities in uterus and adnexa such as uterine myoma and ovarian cyst. Before the delivery, we examined these women's nipples and found no nipple retraction. We performed several breast massages after 32 weeks and affirmed that both groups had no abnormalities on their nipples. Out of the entire sample, who had no difficulty in uterine contraction within an hour after vaginal delivery, 45 women from case group attempted to breast-feed the newborns. The rest of 37 women in control group carried out breast-feeding during 2 hours after and within 24 hours of the delivery.

To assess level of knowledge on breast-feeding, collection of data was carried out in two different stages. 
Surveys contained classifications of the sample by their age, means of infant feeding before the hospital discharge (breast-feeding, milk powder), breast-feeding experience, means of infant feeding (breast, milk powder) after provision of information, length of feeding period, patients' education level and their occupation. These surveys were conducted twice after $1^{\text {st }}$ week and $4^{\text {th }}$ week of birth delivery through telephone interviews. The research data was analyzed using One-way ANOVA and statistical significance is found when $\mathrm{P}$ value are less than $5 \%(p<$ 0.05).

\section{RESULTS}

In this section, we analyzed how infant imprinting through early breast-feeding affects postpartum breast-feeding, and effectiveness of education on breast-feeding. The sample of 82 women were divided into two distinctive groups of one that contained women who began breastfeeding within 2 hours after the delivery, and the other group that began breast-feeding in between 2 hours after and within 24 hours of the delivery. The women who later did combination feeding were 4 after the $1^{\text {st }}$ week and became 8 after the 4 weeks after the delivery. These combination feeders were excluded from observation to divide groups into whole breast milk feeding group and whole power milk feeding group (Table 1). In Table 1, there were some differences of maternal age and neonatal weight between case group and control group. However, no statistical significance was found in $p$-value.

For antepartum examinations, both groups made periodical ambulatory visits and were discharged after 2 days of hospitalization. And for postpartum women in both groups went to postpartum care center. Except for the individual's education level and their means of feeding after information provision, other factors did not show statistically significant differences between the groups. However, although there were no statistically significant differences in maintenance of breast-feeding before hospital discharge $(p=0.53)$, after $1^{\text {st }}$ week $(p=0.40)$, and after $4^{\text {th }}$ week $(p=0.40)$, the tendency of decline in breast-feeding was observed in the control group compared with case group (Table 2).

\subsection{Effects of Education Level on Feeding Types}

In comparing education level of the women and their feeding types (breast, powder milk), statistical signifycance was found in group of women with college degree that these women maintain higher percentage of breastfeeding even 4 weeks after hospital discharge ( $p=0.01$, Table 3). We expect that there is a correlation between one's higher educational level and her financial affluence, and that such kind of correlation was revealed in the case of maintenance of breast-feeding 4 weeks after hospital discharge. Realistically, it would not have been easy for mothers with lower level of education and less financial stability to continue breast-feeding after 4 weeks of the postpartum vacation.

\subsection{Effects of Information Provision on Feeding Types}

Feeding types (breast, powder milk) after information provision display statistically significant differences among the samples only in period before the hospital discharge by $p=0.03$. $p$-value after first week was 0.97 and after four weeks was 0.83 . This implies that major childbirth preparation before delivery is performed through reviewing booklets about feeding types and methods. In this sense, using booklets in breast-feeding education is expected to boost breast-feeding practice, yet consistent education after the delivery is also required (Table 4).

Table 1. Demographic comparison of case groups and control groups in pregnant women and newborns.

\begin{tabular}{cccc}
\hline & Case $(n=45)$ & Control $(n=37)$ & $p$-value \\
\hline Maternal age (mean, year) & 31.3 & 29.8 & 0.08 \\
Gestational age (mean, weeks) & 38.6 & 39.1 & 0.30 \\
Neonatal weight (mean, grams) & 3352 & 3238 & 0.06 \\
Apgar score at 1 m $^{*}$ more than 7 & 45 & 37 & 0.43 \\
Apgar score at 5 m $^{*}$ more than 7 & 45 & 37 & 0.24 \\
\hline
\end{tabular}

Table 2. Feeding types in both groups.

\begin{tabular}{|c|c|c|c|c|c|c|c|}
\hline & & \multicolumn{2}{|c|}{ Case } & \multicolumn{2}{|c|}{ Control } & \multirow{2}{*}{ Total } & \multirow{2}{*}{$p$-vaule } \\
\hline & & Breast feeding & Powdered milk & Breast feeding & Powdered milk & & \\
\hline \multirow{2}{*}{ Feeding types during hospitalization } & $\mathrm{N}$ & 40 & 5 & 6 & 31 & 82 & \multirow{2}{*}{0.53} \\
\hline & $\%$ & $48.8 \%$ & $6.1 \%$ & $7.3 \%$ & $37.8 \%$ & $100.0 \%$ & \\
\hline \multirow{2}{*}{ Feeding types 1 week after delivery } & $\mathrm{N}$ & 40 & 4 & 6 & 28 & 78 & \multirow{2}{*}{0.32} \\
\hline & $\%$ & $51.3 \%$ & $5.1 \%$ & $7.7 \%$ & $35.9 \%$ & $100.0 \%$ & \\
\hline \multirow{2}{*}{ Feeding types 4 weeks after delivery } & $\mathrm{N}$ & 36 & 8 & 8 & 22 & 74 & \multirow{2}{*}{0.40} \\
\hline & $\%$ & $48.7 \%$ & $10.8 \%$ & $10.8 \%$ & $29.7 \%$ & $100.0 \%$ & \\
\hline \multirow{2}{*}{ Total } & $\mathrm{N}$ & 116 & 17 & 20 & 81 & 234 & \\
\hline & $\%$ & $49.6 \%$ & $7.3 \%$ & $8.5 \%$ & $34.6 \%$ & $100.0 \%$ & \\
\hline
\end{tabular}


Table 3. Level of education and feeding types.

\begin{tabular}{|c|c|c|c|c|c|c|}
\hline & & & \multicolumn{2}{|c|}{ Feeding types during hospitalization } & \multirow{2}{*}{ Total } & \multirow{2}{*}{$p$-vaule } \\
\hline & & & Breast feeding & Powdered milk & & \\
\hline \multirow{6}{*}{ Education level } & \multirow{2}{*}{ Non-graduates } & $\mathrm{N}$ & 33 & 7 & 40 & \multirow{4}{*}{0.54} \\
\hline & & $\%$ & $82.5 \%$ & $17.5 \%$ & $100.0 \%$ & \\
\hline & \multirow{2}{*}{ Graduates } & $\mathrm{N}$ & 38 & 4 & 42 & \\
\hline & & $\%$ & $90.5 \%$ & $9.5 \%$ & $100.0 \%$ & \\
\hline & & & \multicolumn{2}{|c|}{ Feeding types 1 week after delivery } & & \\
\hline & & & Breast feeding & Powdered milk & & \\
\hline \multirow{6}{*}{ Education level } & \multirow{2}{*}{ Non-graduates } & $\mathrm{N}$ & 32 & 5 & 37 & \multirow{4}{*}{0.69} \\
\hline & & $\%$ & $86.5 \%$ & $13.5 \%$ & $100.0 \%$ & \\
\hline & \multirow{2}{*}{ Graduates } & $\mathrm{N}$ & 36 & 5 & 41 & \\
\hline & & $\%$ & $87.8 \%$ & $12.2 \%$ & $100.0 \%$ & \\
\hline & & & \multicolumn{2}{|c|}{ Feeding types 4 weeks after delivery } & & \\
\hline & & & Breast feeding & Powdered milk & & \\
\hline \multirow{4}{*}{ Education level } & \multirow{2}{*}{ Non-graduates } & $\mathrm{N}$ & 25 & 9 & 34 & \multirow{4}{*}{0.01} \\
\hline & & $\%$ & $73.5 \%$ & $26.5 \%$ & $100.0 \%$ & \\
\hline & \multirow{2}{*}{ Graduates } & $\mathrm{N}$ & 33 & 7 & 40 & \\
\hline & & $\%$ & $82.5 \%$ & $17.5 \%$ & $100.0 \%$ & \\
\hline
\end{tabular}

Table 4. Information providers and feeding types.

\begin{tabular}{|c|c|c|c|c|c|c|}
\hline & & & \multicolumn{2}{|c|}{ Feeding types during hospitalization } & \multirow{2}{*}{ Total } & \multirow{2}{*}{$p$-value } \\
\hline & & & Breast feeding & Powdered milk & & \\
\hline \multirow{9}{*}{ Information provider } & booklet & $\mathrm{N}$ & 36 & 2 & 38 & \multirow{9}{*}{0.03} \\
\hline & \multirow{2}{*}{ media } & $\mathrm{N}$ & 29 & 6 & 35 & \\
\hline & & $\%$ & $82.9 \%$ & $17.1 \%$ & $100.0 \%$ & \\
\hline & \multirow{4}{*}{ medical provider } & $\mathrm{N}$ & 1 & 0 & 1 & \\
\hline & & $\%$ & $100.0 \%$ & $0.0 \%$ & $100.0 \%$ & \\
\hline & & $\mathrm{N}$ & 5 & 2 & 7 & \\
\hline & & $\%$ & $71.4 \%$ & $28.6 \%$ & $100.0 \%$ & \\
\hline & \multirow{2}{*}{ acquaintance } & $\mathrm{N}$ & 0 & 1 & 1 & \\
\hline & & $\%$ & $0.0 \%$ & $100.0 \%$ & $100.0 \%$ & \\
\hline & & & \multicolumn{2}{|c|}{ Feeding types 1 week after delivery } & & \\
\hline \multirow{10}{*}{ Information provider } & \multirow{2}{*}{ booklet } & $\mathrm{N}$ & 33 & 5 & 38 & \multirow{8}{*}{0.97} \\
\hline & & $\%$ & $86.8 \%$ & $13.2 \%$ & $100.0 \%$ & \\
\hline & media & $\mathrm{N}$ & 29 & 4 & 33 & \\
\hline & & $\%$ & $87.9 \%$ & $12.1 \%$ & $100.0 \%$ & \\
\hline & family & $\mathrm{N}$ & 1 & 0 & 1 & \\
\hline & 1аппу & $\%$ & $100.0 \%$ & $0.0 \%$ & $100.0 \%$ & \\
\hline & \multirow{2}{*}{ medical provider } & $\mathrm{N}$ & 5 & 1 & 6 & \\
\hline & & $\%$ & $83.3 \%$ & $16.7 \%$ & $100.0 \%$ & \\
\hline & & & \multicolumn{2}{|c|}{ Feeding types 4 weeks after delivery } & & \\
\hline & & & Breast feeding & Powdered milk & & \\
\hline \multirow{6}{*}{ Information provider } & \multirow{2}{*}{ booklet } & $\mathrm{N}$ & 29 & 7 & 36 & \multirow{6}{*}{0.83} \\
\hline & & $\%$ & $80.6 \%$ & $19.4 \%$ & $100.0 \%$ & \\
\hline & family & $\mathrm{N}$ & 1 & 0 & 1 & \\
\hline & & $\%$ & $100.0 \%$ & $0.0 \%$ & $100.0 \%$ & \\
\hline & \multirow{2}{*}{ medical provider } & $\mathrm{N}$ & 4 & 2 & 6 & \\
\hline & & $\%$ & $66.7 \%$ & $33.3 \%$ & $100.0 \%$ & \\
\hline
\end{tabular}




\section{DISCUSSION}

The background of setting breast-feeding period to 2 hours after the delivery extends to previous studies purporting that 2 hours is the minimum time that takes to determine whether there is extra blooding or other complications for women after delivery. Also, it is the minimum time that takes for the newborn to recover its body temperature. Accordingly, we set the earliest time to conduct breast-feeding without causing health concerns for both mothers and the newborns as 2 hours [4]. It is a well-recognized knowledge to breast-feed the newborn within the three days after the delivery [3], and this article attempts to find the earlier point in time for breastfeeding. Other research has reported that education on breast-feeding continues to affect both mothers' and the newborns' future health issues [5].

In this result, no statistical significance was found to support that early breast-feeding after two hours of the delivery does no effect and that early breast-feeding improves imprinting between the mother and the newborn. However, an increase in the rate of whole breast-feeding maintenance reflects that imprinting through early breastfeeding will be eventually helpful to maintenance of breastfeeding. Additionally, as factors like education level of individual mother and prenatal education are shown to play important role in breast-feeding maintenance, education and care about breast-feeding seem integral.

Other research suggested that the factors that affect breast-feeding can be divided into variable and invariable factors. The invariable factors included ethnical background, education level of pregnant women, age, neighborhood, their financial affluence. And, variable factors were participation in prenatal education, nearby hospitals, and help from family members [6].

In this light, in order to maintain the sustainability of breast-feeding, development and provision of diverse educational programs are crucial. According to research under university hospitals and hospitals that advocate breastfeeding, it was found that a patient's decision on breastfeeding choice is likely to follow her hospital recommendation. The research also suggested that the breastfeeding rate declines after a month. They additionally found that the pre-natal and post-natal education about breast-feeding play a potent role in maintaining breastfeeding practice. Thus, breast-feeding education seemed to be one of the critical factors that influence pre-natal and post-natal period [7].

Introducing an educational program that encourages breast-feeding [8] had boosted mothers' confidence in breast-feeding and changed their perspective thereafter, realizing that they did not practice enough breast-feeding. In this manner, to encourage consistent practice of breastfeeding, developing an effective educational program was important. This study has demonstrated that conducting breast-feeding within 2 hours after the delivery positively affects continuation of breast-feeding, if no complications are found in both mother and the newborn. Thus, breast-feeding care program which specifies concept of time should be developed.

According to some research, simple knowledge about breast-feeding did not transform into actual breast-feeding practice while physical disability such as nipple or breast milk amount affect breast-feeding practice [9]. In addition to the importance of education in breast-feeding practice, nipple care also requires critical attention. Moreover, in regards of an association with postnatal depresssion, mothers who did not go through postnatal depresssion actively practiced breast-feeding [10]. Thus, providing care to postnatal emotional disruptions was necessary. In this research, it was found that hospital's provision of breast-feeding information was effective in encouraging breast-feeding until 7 days after the program, yet its effectiveness was lost after 4 weeks. This indicated that educational program about breast-feeding practice needs to be carried out at regular basis. Also, as high breast-feeding rate was observed in the pool of mothers with good conditioned nipples, breast care before and after the pregnancy was another crucial factor to breastfeeding. Moreover, although educational level of individuals did not display particular difference in feeding types after first week of the delivery, it demonstrated statistically significant differences after four weeks. No significant difference in feeding types is observed in the first week because most women experience milk fever regardless of their academic achievement levels. On the other hand, for the consistent breast-feeding as long as 4 weeks requires acknowledging importance of breastfeeding, and thus, individuals' education level seem to result in significant difference. This implies that consistent educational program and breast care at regular basis positively affect maintenance of breast-feeding.

As above results have demonstrated, early breast-feeding seemed to contribute in increasing the rate of whole breast-feeding maintenance while prenatal breast-feeding education sustained breast-feeding rate, helped understanding and promoted its practice. However, this research was conducted by a single institution and results in a few limitations. To begin with, consistent observation after four weeks from the delivery to check breastfeeding maintenance was absent. Also, the women under this study were constituted of ones who experienced vaginal delivery. Women with preterm delivery and other complications were not taken into account. Thus, further studies that encompass greater variety and number of women in delivery are required to determine association between prenatal breast-feeding education and maintenance of breast-feeding practice. 


\section{CONCLUSION}

Early breast-feeding seemed to contribute in increasing the rate of whole breast-feeding maintenance while prenatal breast-feeding education sustained breast-feeding rate, helped understanding and promoted its practice. However, this research was conducted by a single institution and had a few limitations. Thus, further studies that encompass greater variety and number of women in delivery are required to determine association between prenatal breast-feeding education and maintenance of breast-feeding practice.

\section{ACKNOWLEDGEMENTS}

The authors appreciate Ms. Sun-Kyung Park, R.N. in director of Quality Improvement team for her help in statistical analysis of collected data. We also wish to sincerely thank Eun-Ae Jung and Eun-Hee Kim for their assistance in manuscript editing.

\section{REFERENCES}

[1] Bellamy C. (UNICEF) (1999) The state of the world's children 1999: Education. UNICEF, New York, 1999.

[2] Seo, J.W., Kim, Y.J., Lee, K.H., Kim, J.Y., Sim, J.G., Kim, H.S., et al. (2002) A survey on the understanding of breast-feeding in pregnant woman. Journal of the Korean Pediatric Society, 45, 575-587.
[3] Korean Society of Obstetrics Gynecology (2007) Obstetrics. 4th Edition, Koonja Publishing Inc., Seoul.

[4] Han, J.S. and Park, I.S. (1993) A study on the process of the newborns' recovering normal temperature just after arriving at the nursery. Chungnam Medical Journal, 20, 679-691.

[5] Kim, T.H. (2009) Breast care of obstetrical patients. Journal of Soonchunhyang Medical Science, 14, 85-88.

[6] Gray-Donald, K., Kramer, M.S., Munday, S. and Leduc, D.G. (1985) Effect of formula supplementation in the hospital on the duration of breast-feeding: A controlled clinical trial. Pediatrics, 75, 514-518.

[7] Park, J.H., Park, Y.G., Jun, H.R., Park, S.W., Lee, J.Y., Hwang, S.G., et al. (1999) Impact of delivery method and feeding supervision on breastfeeding. Journal of the Korean Society of Maternal and Child Health, 3, 7-21.

[8] Suh, Y.S. and Lee, E.S. (1998) The effect of breast feeding promotion program on the changes in confidence towards the breast feeding practice. Journal of the Korean Society of Maternal and Child Health, 2, 13-27.

[9] Eum, G.R., Sohn, H.S. and Kim, H.Y. (2007) Breast feeding rate and related factors in Busan. Journal of the Korean Society of Maternal and Child Health, 11, 78-85.

[10] Ryu, A.L., Kim, T.H. and Lee, H.H. (2010) Evaluation of risk factors for the postpartum depression with Edinburgh Postnatal Depression Scale (EPDS) score. Korean Journal of Perinatology, 21, 74-80. 